\title{
ARTíCULO
}

\section{Biomasa y abundancia de Copepoda (Crustacea) en aguas superficiales del océano Pacífico colombiano durante septiembre de 2007}

Biomass and abundance of Copepoda (Crustacea) in surface waters of the Colombian Pacific Ocean during September, 2007

\author{
Juan C. Jaimes ${ }^{1}$ y Raúl H. López ${ }^{1}$ \\ ${ }^{1}$ Universidad Militar 'Nueva Granada’, Facultad de Ciencias, Programa de Biología Aplicada, Grupo de Investigación en \\ Hidrobiología Aplicada (HIDROBIA), Carrera 100 No 101-80; Bogotá D.C. Colombia. juan.jaimes@unimilitar.edu.co, \\ raul.lopez@unimilitar.edu.co
}

\begin{abstract}
Twenty-three mesozooplankton samples were taken by oblique tows through the $\sim 160 \mathrm{~m}$ with a bongo net during September 2007 in the Colombian Pacific Ocean (OPC). The spatio-temporal variation and oceanographic conditions effects on the abundance and volumetric biomass variation of 5 size classes of copepods were evaluated. Temperature, salinity, dissolved oxygen and chlorophyll- $a$, per station, and their possible relation to the biomass and abundance of those organisms were analyzed. The water column was characterized by a mixed layer of $c a$., $30 \mathrm{~m}$, a thermocline around $80 \mathrm{~m}$ and a bottom layer of cold water $\left(17.4 \pm 0.5^{\circ} \mathrm{C}\right)$ and low oxygen concentration $\left(1.36 \pm 0.2 \mathrm{mg} \mathrm{L}^{-1}\right)$. This stratification may have favored the retention of small-size copepods in the mixed layer. Furthermore, the presence of large size copepods in surface waters could be due to migratory behavior in the water column. The abundance reached 14,933 ind. $100 \mathrm{~m}^{-3}(1,641$ $\mathrm{mm}^{3} 100 \mathrm{~m}^{-3}$ ), where $79 \%$ was composed of copepods with cephalothorax length $<1 \mathrm{~mm}$. Their homogeneous distribution could be linked to the physico-chemical conditions of the surface layer in all the study areas. There was a strong correlation among the higher abundances and biomasses with waters with higher density and low concentration of chlorophyll- $a$.
\end{abstract}

Key words: Holoplankton, microcrustaceans, mesotrophy, mixed layer

Resumen.- Se recolectaron 23 muestras de zooplancton mediante arrastres oblicuos hasta $160 \mathrm{~m}$ con una red bongo durante septiembre de 2007 en el océano Pacífico colombiano (OPC). Se evaluó el efecto nictimeral y las condiciones oceanográficas sobre las variaciones espaciales y temporales de la abundancia y biomasa volumétrica de 5 clases de tamaño de copépodos. También se analizó la temperatura, la salinidad, el oxígeno disuelto y la clorofila- $a$, por estación, y su posible relación con la biomasa y la abundancia de estos organismos. La columna de agua se caracterizó por una capa de mezcla de aprox. $30 \mathrm{~m}$, una termoclina alrededor de $80 \mathrm{~m}$ y una capa inferior de agua fría $\left(17,4 \pm 0,5^{\circ} \mathrm{C}\right)$ y de baja concentración de oxígeno $\left(1,36 \pm 0,2 \mathrm{mg} \mathrm{L}^{-1}\right)$. Esta estratificación pudo haber favorecido la permanencia de los copépodos de tamaños pequeños en la capa de mezcla. Además, la presencia de copépodos de tamaños grandes en aguas superficiales pudo deberse a comportamientos migratorios en la columna de agua. La abundancia general alcanzó 14.933 ind. $100 \mathrm{~m}^{-3}$ $\left(1.641 \mathrm{~mm}^{3} 100 \mathrm{~m}^{-3}\right)$, donde el $79 \%$ se compuso de copépodos de longitud cefalotoráxica < $1 \mathrm{~mm}$, cuya distribución homogénea entre las estaciones podría relacionarse con las condiciones físico-químicas de la capa superficial en todas las áreas de estudio. Se observó una fuerte correlación entre las altas abundancias y biomasas con aguas de mayor densidad y baja concentración de clorofila- $a$.

Palabras clave: Holoplancton, microcrustáceos, mesotrofia, capa de mezcla

\section{INTRODUCCIÓN}

El Orden Copepoda es uno de los grupos de crustáceos más diverso y adaptado a gran variedad de ambientes; por lo general, representa el mayor porcentaje de la biomasa zooplanctónica (Paffenhöfer \& Mazzocchi1 2003, Márquez et al. 2009), siendo muy relevante en la ecología trófica de larvas de peces, crustáceos (Lavens \& Sorgeloos
1996, Siordia et al. 2006, Landaeta et al. 2011) y organismos gelatinosos carnívoros (Vergara et al. 2010), cumpliendo un papel importante en el flujo vertical de carbono (Lavaniegos 2007, Cass 2011). De presencia amplia y ligada a condiciones oceanográficas (Lavaniegos \& González 1999, López-Ibarra \& Palomares-García 2006, Aceves- 
Medina et al. 2007), la variación en su abundancia puede igualmente relacionarse con frentes oceánicos y surgencias, así como a la ontogenia o comportamiento trófico propio de los copépodos (Zalkina 1970, Cervantes et al. 1993, López-Ibarra \& Palomares-García 2006). De igual manera, se destaca su importancia en el desarrollo de técnicas de larvicultura (Velázquez et al. 2001, Forrnes et al. 2010).

Rodríguez (2005) resalta la importancia de la biomasa de copépodos distribuida por clase de tamaños en ecosistemas pelágicos, lo que ayuda a caracterizar el flujo energético. Woodward et al. (2005) y Woodward \& Warren (2007) resaltan el papel principal del tamaño de los copépodos y mencionan la importancia de incorporar este aspecto en modelos teóricos que ayuda a explicar la estabilidad de la trama trófica, los flujos de energía y las respuestas a las perturbaciones; también señalan que el tamaño ayuda a entender la estructura y funcionamiento de las redes tróficas y las consecuencias probables del cambio en la biodiversidad.

En Colombia, tanto en aguas marinas como continentales, se han realizado diversos estudios sobre copépodos enfocados a parámetros poblacionales (Atencio et al. 2005, Gaviria \& Aranguren 2007, Martínez-
Barragán et al. 2009), taxonómicos (Giraldo \& Gutiérrez 2007, Medellín-Mora \& Navas 2010), fisiológicos (Ramírez et al. 2005), o bien parte de estudios de comunidades zooplanctónicas (Bernal \& Zea 2000, Giraldo et al. 2011). No hay información relacionada con la distribución por tamaños de copépodos y su posible efecto dentro del flujo energético. Teniendo en cuenta la relevancia del tamaño de los organismos en las redes tróficas y la escasa información de este aspecto en aguas del Pacífico colombiano, este trabajo reseña el efecto de las condiciones oceanográficas y nictimerales sobre las variaciones espaciales y temporales de la abundancia y biomasa volumétrica de 5 clases de tamaño de copépodos en aguas del océano Pacífico colombiano (OPC) durante septiembre de 2007, en un crucero oceanográfico realizado dentro del Estudio Regional del Fenómeno El Niño (ERFEN).

\section{MATERIALES Y MÉTODOS}

\section{ÁreA DE ESTUDIO}

El OPC se ubica entre las coordenadas $01^{\circ} 30^{\prime \prime}-07^{\circ} 10^{\prime \prime} \mathrm{N}$ y $77^{\circ} 40^{\prime \prime}-82^{\circ} 00^{\prime \prime O}$ (Fig. 1). Climáticamente, está influido por los desplazamientos norte-sur de la Zona de
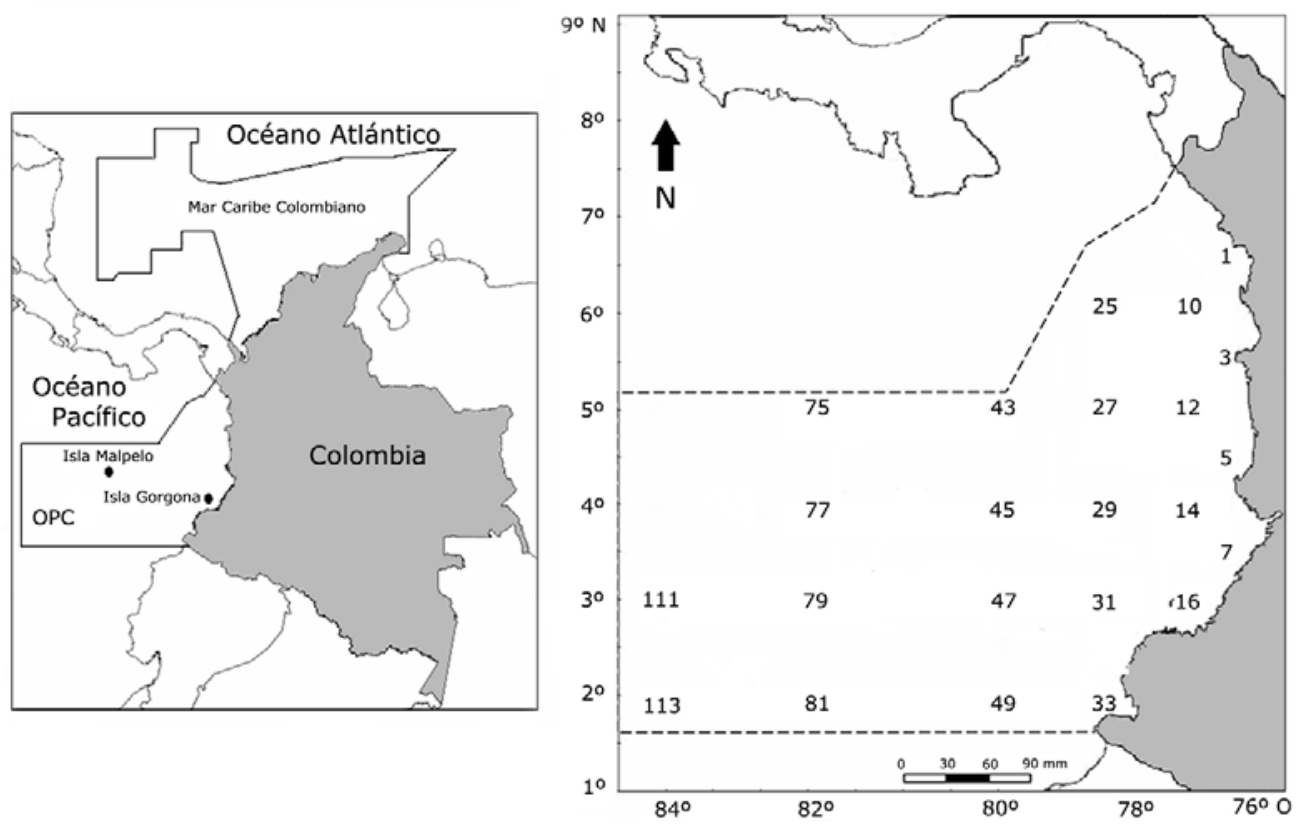

Figura 1. Mapa del área de estudio mostrando la grilla de estaciones muestreadas (numeración del ERFEN) / Map of the study area showing the grid of stations sampled (ERFEN numbering) 
Convergencia Intertropical (ZCIT) y El Niño-Oscilación del Sur (ENOS) (Villegas \& Málikov 2006), que influyen en las características hidrológicas del OPC y causa variaciones espacio temporales en las características fisicoquímicas en la capa superficial que puede alcanzar los $500 \mathrm{~m}$ de profundidad, de tal forma que alrededor de los $20 \mathrm{~m}$ suele presentarse la termoclina separando una capa superior homogénea de aguas intermedias y profunda. En época seca (marzo) la capa superior es más fría y menos profunda, mientras que en época húmeda (agosto-septiembre), es más cálida y profunda. A lo largo del año son comunes los eventos de surgencia de diferente intensidad y amplitud en casi toda el área (CCCP 2002, Fiedler \& Talley 2006, Clarke 2008) (Fig. 1).

\section{CAMPAÑA OCEANOGRÁFICA}

Los muestreos se realizaron durante el crucero oceanográfico de la serie ERFEN, que tuvo lugar entre el 4 y 28 de septiembre 2007, en 23 estaciones distribuidas en todo el OPC (Fig. 1). Los arrastres de zooplancton fueron oblicuos, hasta una profundidad promedio de 184 \pm 42 m, filtrando $207 \pm 57$ L estación ${ }^{-1}$. Se empleó una red bongo con mallas de 294 y $520 \mu \mathrm{m}$, diámetro de $60 \mathrm{~cm}$ y longitud de 1,85 m. Para determinar el volumen de agua filtrada se utilizó un flujómetro digital Hydro-Bios. Las muestras se fijaron con formaldehído al 10\% en agua de mar. En cada estación se registró la posición geográfica, la hora de muestreo y el periodo lunar. Se empleó una sonda CTD (Conductividad, Temperatura, Densidad) Sea Bird Electronics 19 para medir la salinidad y la temperatura en la columna de agua cada 20 m los cuales fueron graficados, y un termómetro de cazoleta para medir la temperatura en la superficie. Con botellas Niskin de $5 \mathrm{~L}$ se extrajeron muestras de agua para determinar la concentración de clorofila- $a$ estimada mediante el método de extracción con acetona (90\%) y cuantificación por espectrofotometría (Clesceri et al. 2001).

\section{Biomasa y AbUndancia}

En este estudio se utilizaron muestras obtenidas con red de poro de $294 \mu \mathrm{m}$. Para determinar la biomasa volumétrica de los copépodos, se establecieron 5 rangos de longitud del cefalotórax (Lc) teniendo en cuenta su fácil separación en una grilla de $0,5 \mathrm{~mm}^{2}$, los individuos utilizados para estimar la biomasa volumétrica, fueron seleccionados de las muestras obtenidas tras las divisiones Folsom, separando de la muestra el 1\% para los rangos 0,50-1,00 mm, 1,10-2,00 mm Lc, y el 10\% para los rangos siguientes (Tabla 1). El volumen fue estimado mediante la comparación de las formas geométricas elipsoideesferoide, cilíndrica y cónica con el cefalotórax (prosoma), abdomen (urosoma) y antenas, respectivamente (Fig. 2). Las medidas fueron tomadas a individuos bien preservados, con la ayuda de un microscopio E4-Z10 con escala milimetrada de $0,01 \mathrm{~mm}$ de precisión. El conteo, por rango de longitud del cefalotórax se realizó empleando un estereoscopio Carl Zeiss ${ }^{\circledR}$ Stemi DV4 para cada una de las submuestras por estación obtenidas mediante un divisor Folsom.

Tabla 1. Longitud del cefalotórax (Lc) (mm), Longitud abdominal (La), promedio Lc (PLc) y promedio La (PLa) (mm), y volumen (V) $\left(\mathrm{mm}^{3}\right)$, estimado para cada rango de copépodos; desviación estándar (d.e) / Cephalothorax length (Lc) ( $\mathrm{mm}$ ), abdominal length (La), mean Lc (PIc) and mean La (PLa) (mm), and volume (V) $\left(\mathrm{mm}^{3}\right)$ estimated for each range of copepod; standard deviation (d.e)

\begin{tabular}{cccccccc}
\hline $\mathrm{n}$ & $\mathrm{Lc}$ & PLc & d.e & PLa & d.e & V & d.e \\
\hline 40 & $0,50-1,00$ & 0,81 & 0,15 & 0,31 & 0,11 & 0,05 & 0,03 \\
35 & $1,01-2,00$ & 1,65 & 0,37 & 0,49 & 0,21 & 0,35 & 0,24 \\
39 & $2,01-3,00$ & 2,45 & 0,29 & 0,54 & 0,20 & 0,77 & 0,41 \\
17 & $3,01-4,00$ & 3,63 & 0,26 & 0,55 & 0,07 & 1,83 & 0,48 \\
15 & $4,01-6,00$ & 4,56 & 0,76 & 0,64 & 1,00 & 4,26 & 3,21 \\
\hline
\end{tabular}

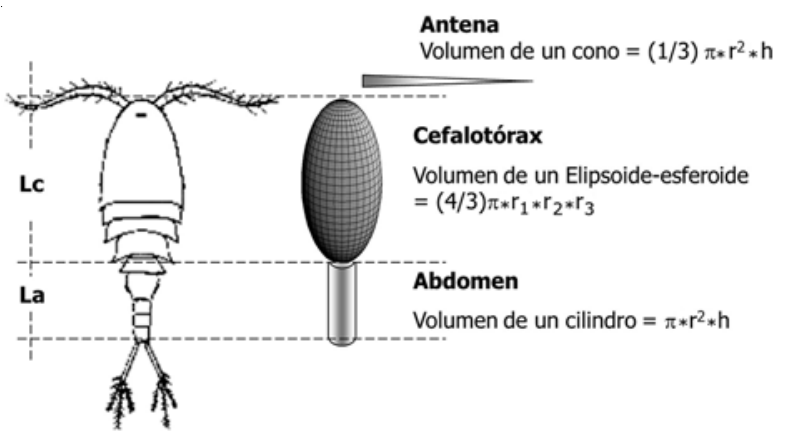

Figura 2. Esquema general de un copépodo y figuras geométricas adoptadas para la estimación volumétrica. Lc= Longitud del cefalotórax, La= Longitud del abdomen / General scheme of a copepod and geometric figures adopted for the volumetric estimation. $\mathrm{Lc}=$ cephalothorax length, $\mathrm{La}=$ abdominal length

\section{Tratamiento DE LOS DATOS}

Las abundancias se estandarizaron según la fórmula:

$$
N=\frac{100 \mathrm{~m}^{3} \cdot \mathrm{n}}{\pi \cdot r^{2} \mathrm{~d}}
$$

donde: $\mathrm{N}$ = Número de individuos $100 \mathrm{~m}^{-3}, \mathrm{n}=$ Número de individuos en cada arrastre, $\mathrm{r}^{2} \mathrm{~d}=$ Volumen de agua filtrada 
en cada arrastre, $\mathrm{r}=$ Radio de la red, $\mathrm{d}=l * \cos (\tan -1 * \tan \mathrm{P})$ = profundidad máxima del lance; coseno y tangente referidas al ángulo del cable, $l$ = longitud máxima (m) del cable filado y tan $\mathrm{P}=$ tangente promedio del ángulo del cable, medido cada 30 s (Smith \& Richardson 1979).

Las muestras de zooplancton obtenidas de 6:00 a.m. a 6:00 p.m., se designaron como diurnas y de 6:01 p.m. a 5:59 a.m., como nocturnas. En los análisis se incluyeron las fases lunares básicas: luna llena, cuarto menguante, luna nueva y cuarto creciente.

Se elaboraron perfiles de temperatura para determinar la profundidad de la termoclina, salinidad, clorofila- $a$ y oxígeno disuelto.

Con el fin de determinar la posible relación de la abundancia y la biomasa con la distribución espacial, se aplicó la prueba de Kruskal-Wallis entre las estaciones. De igual forma se aplicó esta prueba para determinar las posibles diferencias en la concentración de clorofila- $a$ y biomasa respecto a las variaciones día-noche, Posteriormente, se realizó un Análisis de Componentes Principales (ACP) entre las variables abióticas (valores brutos de la capa de mezcla) y bióticas de cada uno de los rangos de tamaño. En estos análisis se empleó el paquete Statistica ${ }^{\circledR}$ versión 5.1

\section{Resultados}

Salvo las estaciones 49 y 81, donde la termoclina se ubicó entre los 50 y 70 m de profundidad, en la columna de agua muestreada la capa de mezcla abarcó los primeros $30 \mathrm{~m}$ de profundidad sin presentar diferencias significativas en los valores de salinidad, oxígeno y clorofila- $a$ entre las estaciones ( $\mathrm{t}=13,5, P=0,91$, confiabilidad 95\%) (Fig. 3). Esta capa de mezcla se caracterizó por una temperatura media de $25,1 \pm 0,4^{\circ} \mathrm{C}$, salinidad de $31,6 \pm 1,0$, oxígeno disuelto $6,3 \pm 0,2 \mathrm{mg} \mathrm{L}^{-1}$ y $0,43 \pm 0,1 \mathrm{mg} \mathrm{m}^{-3}$ de clorofila- $a$, con su valor más bajo $\left(0,28 \pm 0,1 \mathrm{mg} \mathrm{m}^{-3}\right)$ en la superficie y su máximo $\left(0,57 \pm 0,6 \mathrm{mg} \mathrm{m}^{-3}\right)$ a $20 \mathrm{~m}$ de profundidad. La termoclina de agua más fría y nivel de oxígeno más bajo (19,2 $\pm 0,4^{\circ} \mathrm{C}, 2,3 \pm 0,7 \mathrm{mg} \mathrm{L}^{-1}$, respectivamente), presentó un aumento en los niveles de clorofila- $a$ y salinidad de $0,5 \mathrm{mg} \mathrm{m}^{-3}$ y 31,6 $\pm 1,0$ respectivamente. A más de $80 \mathrm{~m}$ de profundidad, los valores de temperatura $\left(17,4 \pm 0,5^{\circ} \mathrm{C}\right)$, oxígeno $\left(1,36 \pm 0,2 \mathrm{mg} \mathrm{L}^{-1}\right)$ y clorofila- $a\left(0,16 \pm 0,06 \mathrm{mg} \mathrm{m}^{-3}\right)$ fueron los más bajos, contrarios a la salinidad, pero todos relativamente estables en cuanto aumentó la profundidad.

La capa de mezcla se caracterizó igualmente por presentar un gradiente de temperatura en sentido costa océano y fluctuó entre $27,5^{\circ} \mathrm{C}$ y $23,6^{\circ} \mathrm{C}$. Respecto a la salinidad, su tendencia fue inversa a la temperatura y fluctuó entre 28,0 y 33,6 (Fig. 4). El oxígeno disuelto alcanzó sus valores más bajos hacia la zona oceánica norte, y los más altos hacia la zona centro sur y oriente con rangos entre 7,1 y 4,5 $\mathrm{mg} \mathrm{L}^{-1}$, respectivamente. La clorofila- $a$ presentó las concentraciones más altas hacia el norte oceánico y sur-oriente costero del OPC (Fig. 4).
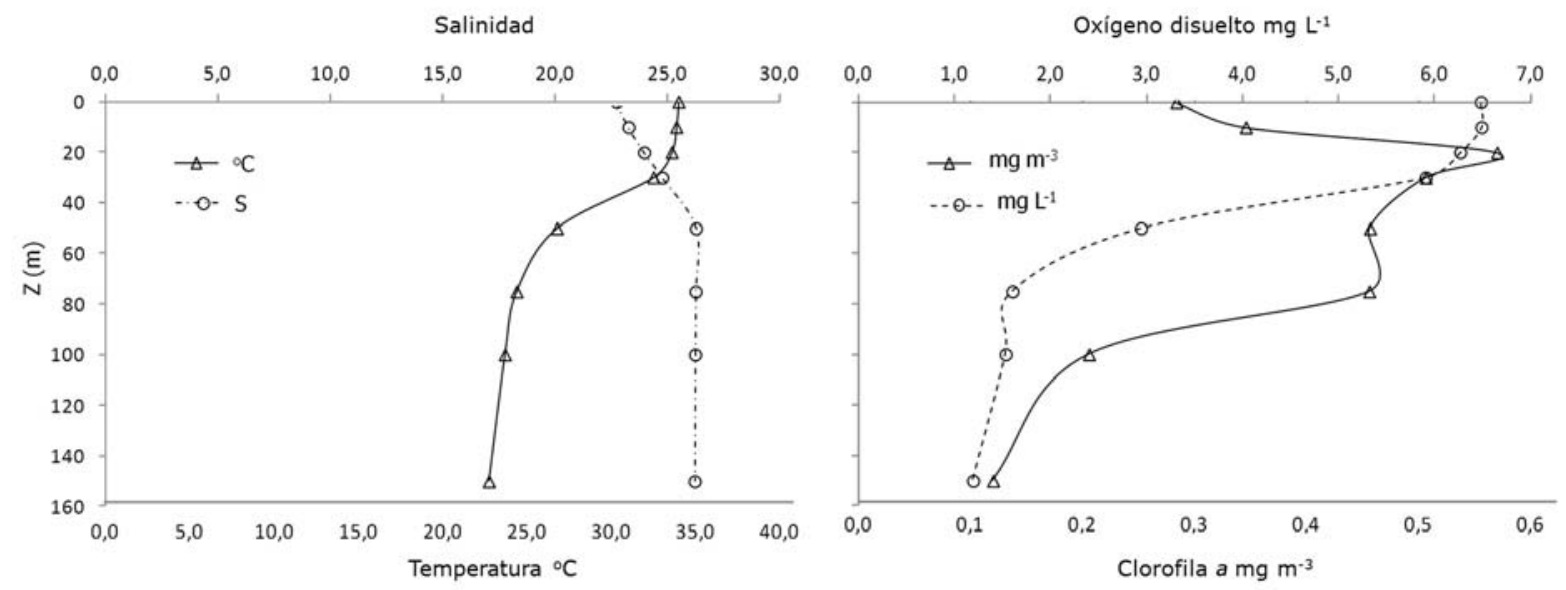

Figura 3. Perfiles del promedio general de salinidad vs. temperatura y oxígeno disuelto vs. clorofila- $a$ en el océano Pacífico colombiano, septiembre 2007 / Profiles of general mean salinity vs. temperature and dissolved oxygen vs. chlorophyll- $a$ in the Colombian Pacific Ocean, September 2007 

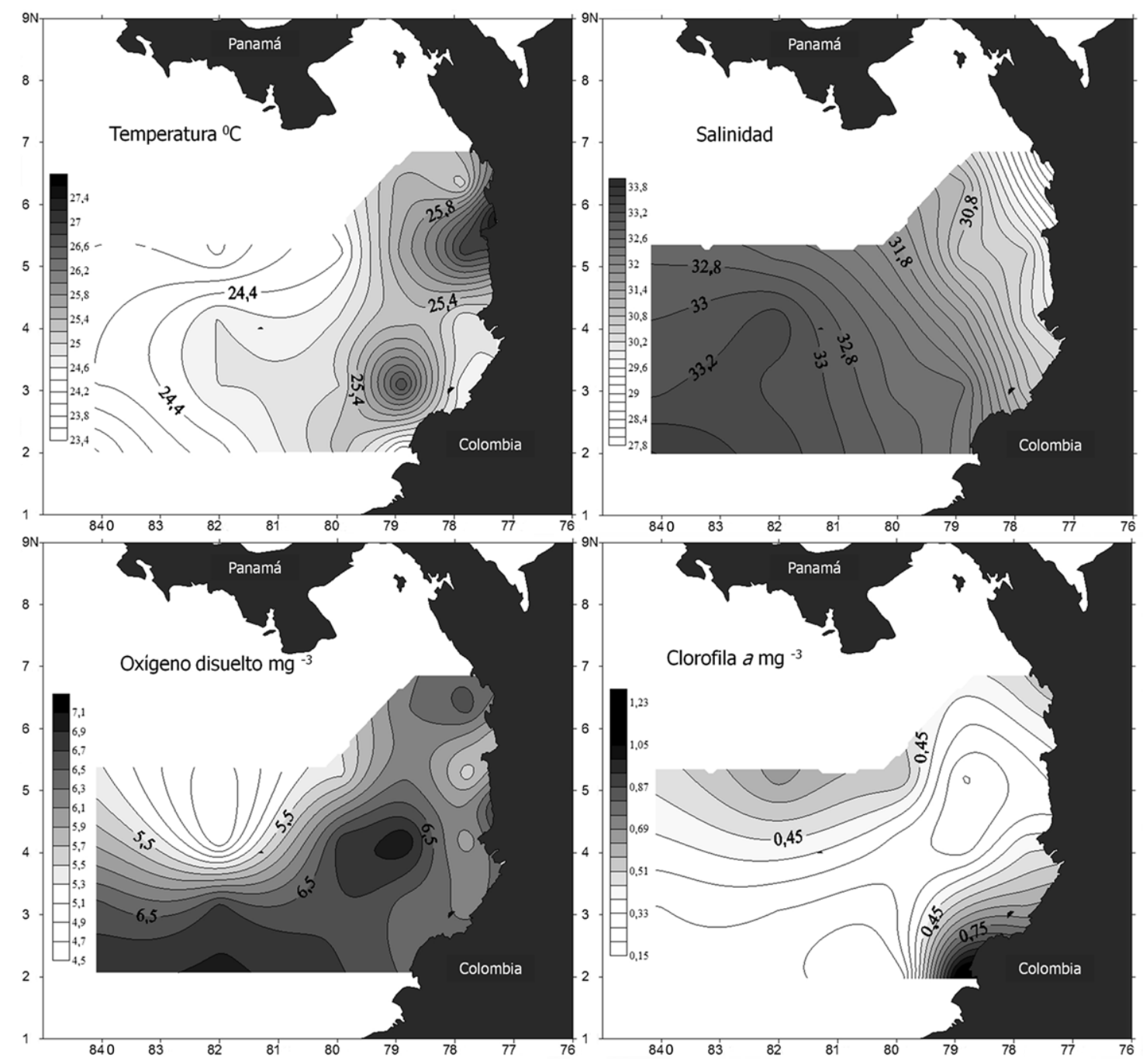

Figura 4. Distribución horizontal de temperatura, salinidad, oxígeno disuelto y clorofila-a promedio en la capa de mezcla en el Océano Pacífico colombiano, septiembre 2007 / Mean temperature, salinity, dissolved oxygen, and chlorophyll-a horizontal distribution in the mixed layer in the Colombian Pacific Ocean, September 2007

Entre los periodos día-noche las variaciones del oxígeno disuelto, clorofila- $a$ temperatura y salinidad, en la columna de agua, no fueron significativamente diferentes ( $\mathrm{t}=1,26, P=0,26$, confiabilidad 95\%). En el caso del oxígeno disuelto se detectó un ligero descenso diurno a los $10 \mathrm{~m}$, mientras que la clorofila- $a$ presentó los valores más altos a los $75 \mathrm{~m}$ en el día y a los $20 \mathrm{~m}$ en la noche (Fig. 5).

Se estimó en promedio una abundancia de copépodos de 14.933 ind. $100 \mathrm{~m}^{-3}$, que correspondió a una biomasa volumétrica de $1.641 \mathrm{~mm}^{3} 100 \mathrm{~m}^{-3}$. La abundancia más baja se estimó en 5.068 ind. $100 \mathrm{~m}^{-3}$, correspondiente a 609 $\mathrm{mm}^{3} 100 \mathrm{~m}^{-3}$ en la estación 75 y la más alta en 36.779 ind.
$100 \mathrm{~m}^{-3}$, correspondiente a $3.952 \mathrm{~mm}^{3} 100 \mathrm{~m}^{-3}$ en la estación 111 , sin diferencias significativas entre estaciones $(t=$ 4,26, $P=0,99$, confiabilidad 95\%). El 79\% de la abundancia se compuso por copépodos con tamaño $<1 \mathrm{~mm} \mathrm{Lc}$, mientras que el $18 \%$ estuvo constituido por individuos entre 1,01 y 2,00 mm Lc, y sólo el 3\% tuvo tamaños $>2$ mm Lc. El mayor aporte de biomasa fue dado por copépodos $<1 \mathrm{~mm}$ Lc (40\%), entre 1,01 y 2,00 mm Lc (40\%) y entre 2,01 y 3,00 mm Lc (15\%), los cuales se distribuyeron ampliamente. Tamaños superiores a los $3 \mathrm{~mm}$ Lc aportaron el $5 \%$ en biomasa con una distribución más restringida (Fig. 6). 


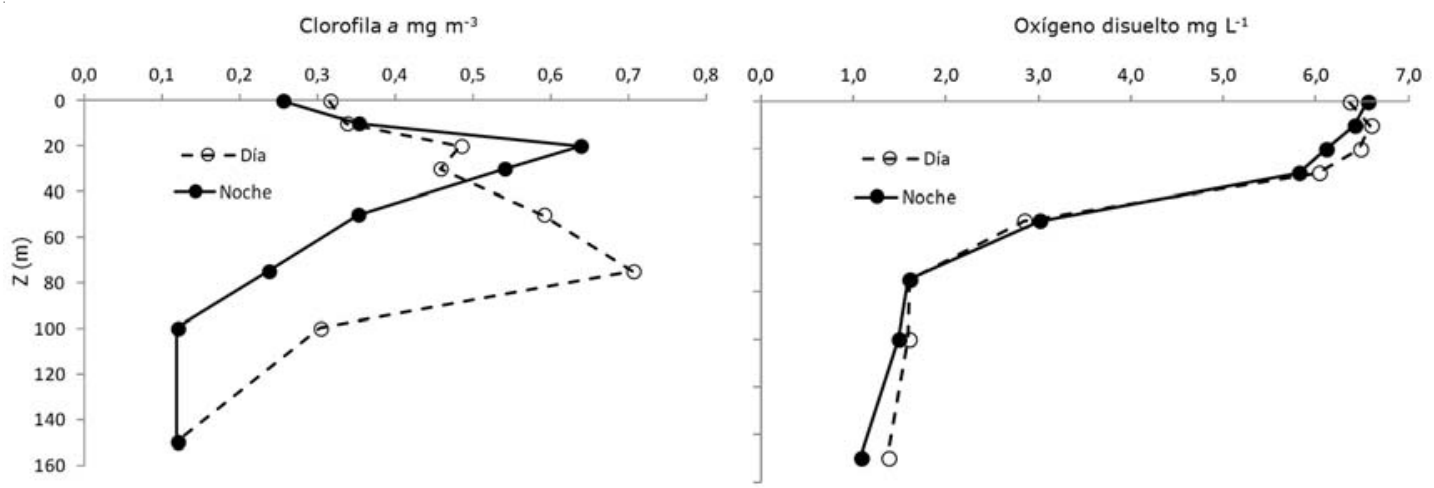

Figura 5. Perfiles del promedio general del oxígeno disuelto y la clorofila- $a$ durante los periodos día-noche en el océano Pacífico colombiano, septiembre 2007 / Profiles of the general mean dissolved oxygen and chlorophyll- $a$ during the day-night periods in the Colombian Pacific Ocean, September 2007

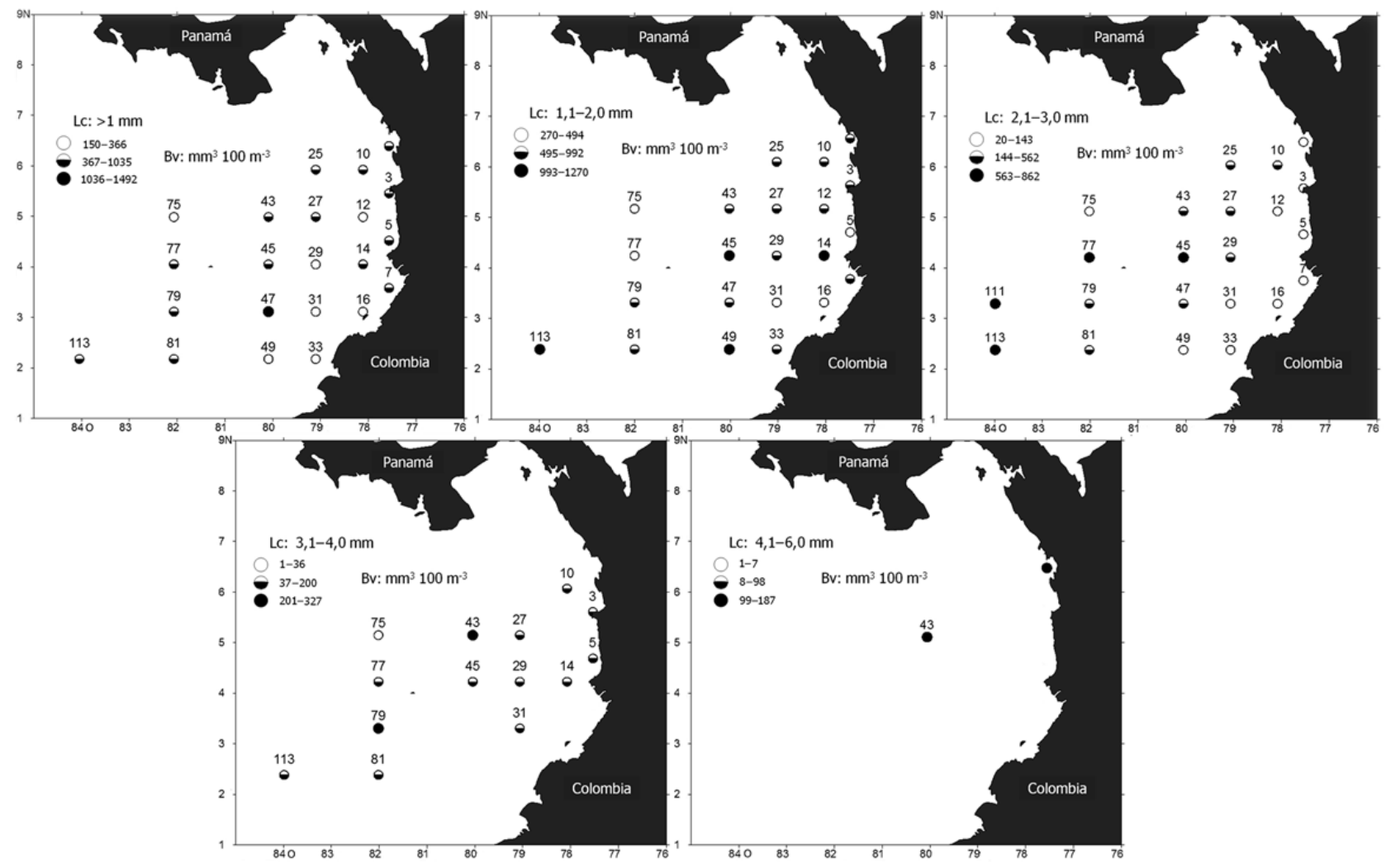

Figura 6. Distribución de los copépodos por rangos de longitud del cefalotórax (Lc) y biomasa volumétrica (Bv) en el océano Pacífico colombiano, septiembre 2007 / Distribution of copepods by ranges of cephalothorax length (LC) and biomass volume (Bv) in the Colombian Pacific Ocean, September 2007 


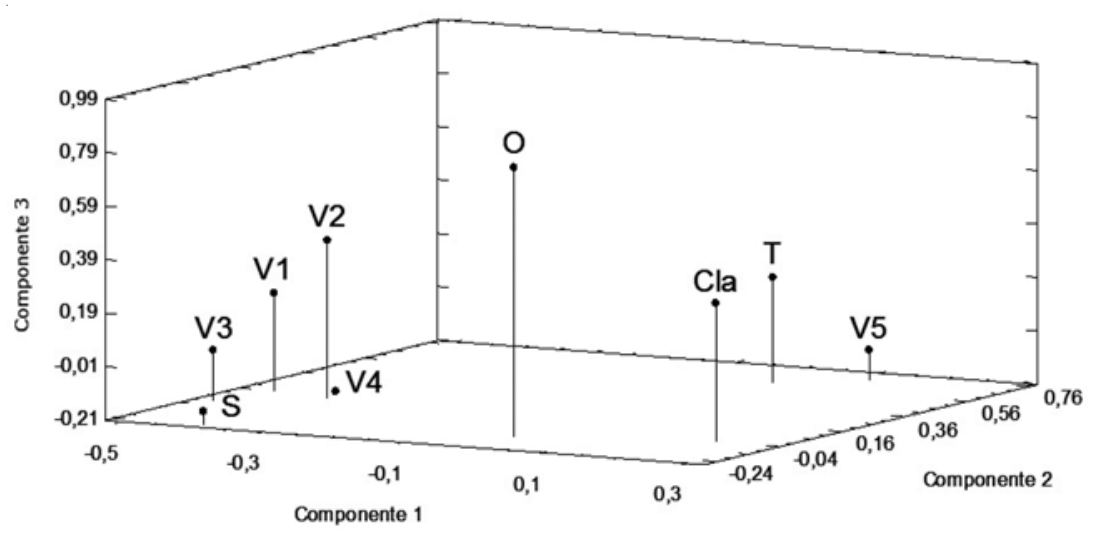

Figura 7. Diagrama ACP entre variables abióticas (S: Salinidad; O: Oxígeno; Cla: Clorofila- $a$; T: Temperatura) y la biomasa volumétrica (V1: $<1 \mathrm{~mm}$; V2: 1,01-2,00 mm; V3: 2,01-3,00 mm; V4: 3,01-4,00 mm y V5: 4,01-6,00 mm Lc), explicando el 66,5\% de la variación / ACP diagram among abiotic variables (S: Salinity; O: Oxygen; Cla: chlorophyll- $a$; T: Temperature) and volumetric biomass (V1: < $1 \mathrm{~mm}$; V2: 1.01-2.00 mm; V3: $2.01-3.00 \mathrm{~mm}$; V4: 3.01-4.00 mm and V5: $4.01-6.00 \mathrm{~mm} \mathrm{LC}$ ), explaining $66.5 \%$ of the variation

El ACP sugiere que el 99,9\% de la abundancia y el $99,2 \%$ de la biomasa representados en copépodos con tamaños menores a $4 \mathrm{~mm}$ Lc se correlacionan más con aguas de mayor densidad y baja concentración de clorofila- $a$, y en menor medida con aguas frías de baja concentraciones de oxígeno disuelto, masas de agua que se ubicaron al suroccidente del OPC (Fig. 7). Los copépodos más grandes se correlacionan en gran medida con aguas de mayor temperatura y menor densidad ubicadas al noreste del OPC. Según el ACP, el 66,5\% de la variación es explicada por los 3 primeros componentes principales.

En cuanto a la variación nictimeral, el 59\% de la biomasa volumétrica (representado por los rangos $<1,2,02-3,00 \mathrm{y}$ 3,01-4,00 mm Lc), correspondiente al 82\% de la abundancia estimada, aumentó durante los periodos diurnos, más notable en los copépodos de menor tamaño. Sin embargo, el $40 \%$ de la biomasa (representado por el grupo de copépodos con rangos entre 1,01-2,00 mm Lc y > a 4,01 $\mathrm{mm} \mathrm{Lc}$ ) no mostró alguna tendencia en relación con los periodos de baja o alta luminosidad. Entre los periodos lunares no se evidenciaron cambios significativos en la biomasa ( $\mathrm{t}=2,84, P=0,41)$, aunque en los copépodos $<$ $4,00 \mathrm{~mm}$ Lc hubo una tendencia al aumento durante luna nueva y luna llena (Fig. 8).

\section{Discusión}

En términos generales la columna de agua en el OPC sigue el mismo patrón de distribución mencionado por CCCP (2002), en el cual hay una capa homogénea que se extiende verticalmente pocos metros de la superficie, seguida de una capa más heterogénea que va hasta unos $80 \mathrm{~m}$ de profundidad y que inicia alrededor de los $30 \mathrm{~m}$; posterior a ella se presenta otra masa de agua más homogénea más fría y de menor salinidad. Si bien Villegas \& Málikov (2006) mencionan que la estructura vertical del OPC presenta 3 capas, de 0 a $80 \mathrm{~m}, 80$ a $200 \mathrm{~m}$ y mayor a $200 \mathrm{~m}$, estas variaciones pueden estar asociadas a la interacción océano-atmosférica que genera turbulencias y desplazamientos verticales de la termoclina (CCCP 2002), lo que sugiere una alta variabilidad en la dinámica vertical de las aguas superficiales del OPC.

Las características de la capa de mezcla superficial difieren de las reportadas por CCCP (2002) para la misma época del año, en que los valores medios de temperatura y salinidad encontrados fueron más bajos que los rangos habituales. No obstante, la capa de mezcla alcanzó los $30 \mathrm{~m}$ de profundidad. Por otra parte la variación horizontal de estos parámetros presenta la misma tendencia, un gradiente de temperatura del océano a la zona costera y un comportamiento inverso para la salinidad. 

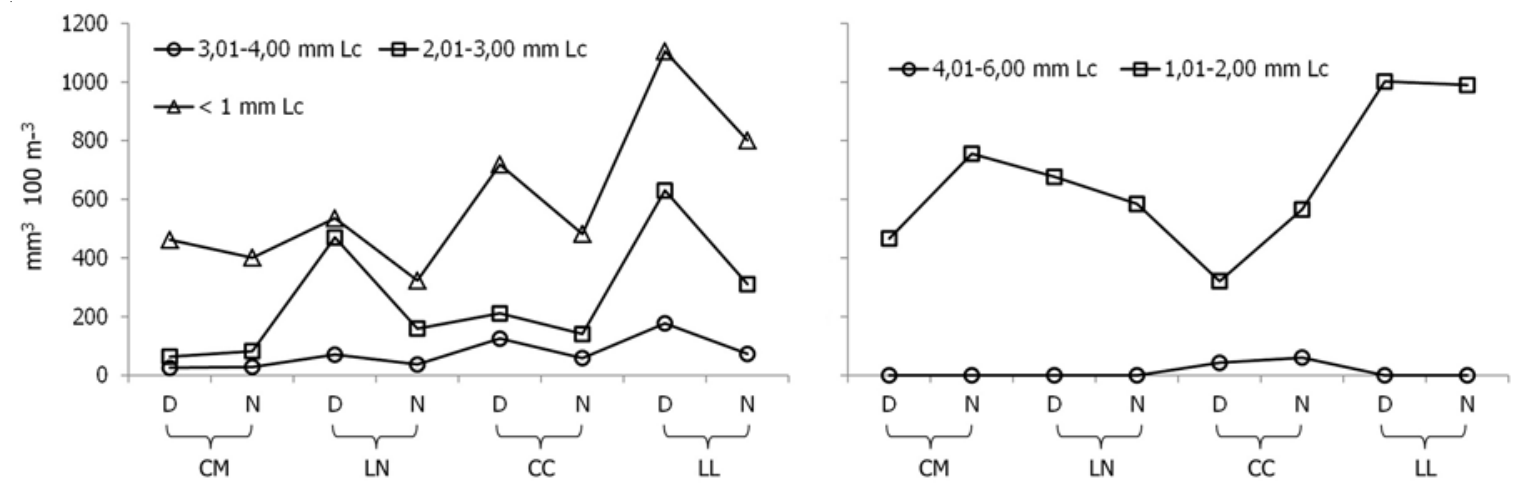

Figura 8. Variación circadiana y lunar de la biomasa volumétrica $\left(\mathrm{mm}^{3} 100 \mathrm{~m}^{-3}\right)$, según los rangos de longitud del cefalotórax (LC) $(\mathrm{mm})$ de los copépodos en el océano Pacífico colombiano, septiembre 2007. CM= Cuarto Menguante, LN= Luna Nueva, CC= Cuarto Creciente, $\mathrm{LL}=$ Luna Llena / Circadian and lunar variation of the volumetric biomass $\left(\mathrm{mm}^{3} 100 \mathrm{~m}^{-3}\right)$ according to the ranges of cephalothorax length (LC) (mm) of copepods in the Colombian Pacific Ocean, September 2007. CM = last quarter, $\mathrm{LN}=$ new moon, $\mathrm{CC}=$ first quarter, $\mathrm{LL}=$ full moon

Teniendo en cuenta la temperatura y salinidad hacia el sur y occidente del OPC (estación 113) se pensaría en eventos de surgencia; no obstante, las bajas concentraciones de oxígeno disuelto y clorofila- $a$, características de estas aguas ascendentes, y los perfiles obtenidos, se sugiere que otro factor como el transporte horizontal de aguas frías fuese el causante de la baja temperatura y salinidad promedio alcanzada durante este periodo en el OPC. CCCP (2002) menciona la existencia de un permanente efecto de surgencia, pero de limitada extensión e intensidad para el segundo periodo del año, cuando, entre julio y septiembre, prevalece un giro anticiclónico en posición central influenciado por la débil corriente del Golfo de Panamá en sentido suroeste (aprox. $200 \mathrm{~km}$ de ancho) y una Corriente de Colombia de mayor intensidad en sentido sur-norte por la zona nerítica (Wooster 1959, Wyrtki 1969, Rodríguez-Rubio \& Schneider 2003). Debido a que no se midieron las corrientes, no se puede determinar su efecto sobre las características descritas. En cuanto a las diferencias horizontales, el aporte de aguas continentales, sería uno de los principales factores que contribuiría al descenso de las concentraciones de sales hacia la zona costera (CCCP 2002, Uribe 2003).

Si bien los arrastres fueron oblicuos y superaron ampliamente la capa de mezcla, es imposible saber con certeza el lugar que ocupaban los copépodos al momento de su captura; se presume que la mayor disponibilidad de biomasa, i.e., copépodos < a $3 \mathrm{~mm}$ Lc (95\%), podrían encontrarse en estos primeros $30 \mathrm{~m}$ de profundidad, ya que la termoclina ejerce una barrera física que limita la distribución de los individuos más pequeños, mientras que los de mayor tamaño superan ampliamente esa capa, como se ha observado desde hace varias décadas en muchas partes del mundo, incluso en el Pacífico oriental tropical (e.g., Greenlanw 1979, Chen 1986, Aridarni 1990, Suárez-Morales 1990, Roman \& Gauzens 1997, Paffenhöfer \& Mazzocchi 2003, Saiz et al. 2003, López et al. 2007, Takahashi \& Uchiyama 2008, Carvajal et al. 2009, Cass 2011).

Las variaciones nictimerales de la biomasa por rango de tamaño exhibieron una tendencia al aumento durante días y noches de luna llena, más marcada en los tamaños pequeños $(<1 \mathrm{~mm} \mathrm{Lc})$, evidenciando un fototropismo positivo bastante fuerte, sumado a una posible mayor tasa de renovación, como lo sugieren Pérez et al. (1987) y Álvarez et al. (2003), y asociado a una menor acción de depredadores durante las horas más luminosas. La tendencia de la disminución de la biomasa y abundancia en horas de baja intensidad lumínica puede estar también asociada a la fotosensibilidad y a la mayor presión de depredadores que pueden consumir pequeños copépodos, larvas de peces, crustáceos y quetognatos (Monsalve 1976, Siordia et al. 2006, Carvajal et al. 2009, López \& Medellín 2010¹), los cuales son muy abundantes en horas de la noche. En este sentido, los copépodos $<2$

${ }^{1}$ López RH \& J Medellín. 2010. Dinámica de la comunidad de copépodos mesozooplanctónicos en el océano Pacífico Colombiano. CD resúmenes XII Congreso Latinoamericano de Ciencias del Mar, 15-19. Abr.07, Florianópolis, Brasil. 
mm Lc están sometidos a una mayor presión depredadora. En relación con el vínculo depredador-presa, Humphries (2007) refiere una relación directa entre el tamaño del depredador y de la presa, aunque, en virtud de la oferta de alimento del tamaño adecuado, puede consumir presas más pequeñas.

Comparado con septiembre de 2003 (Giraldo \& Gutiérrez 2007), el OPC reflejaría una productividad secundaria relativamente baja en septiembre de 2007. Sin embargo, teniendo en cuenta las características en la comunidad de copépodos observada allí, en donde un alto porcentaje está por debajo de los $3 \mathrm{~mm} \mathrm{Lc}$, y las condiciones abióticas como temperatura y concentración de oxígeno disuelto son relativamente altas en la capa de mezcla, se podría decir que existieron condiciones bastante favorables para el desarrollo de etapas tempranas de consumidores secundarios en el OPC.

Turner (2004) menciona que los nauplios, copepoditos y adultos con talla menor al milímetro son los principales componentes de la abundancia del zooplancton y juegan un papel importante como alimento en niveles tróficos más altos, aunque puede llegar a desestimarse esta abundancia, biomasa e impacto del pastoreo, debido al método utilizado, que por lo general son mallas por encima de las $200 \mu \mathrm{m}$ y no capturan esos organismos tan pequeños.

Calbet \& Saiz (2005) resaltan la importancia de la dieta de los copépodos pequeños, que puede incluir fitoplancton minúsculo, pero principalmente protistas heterótrofos, ciliados y dinoflagelados heterótrofos, o son detritívoros de macro-agregados orgánicos, lo que los convierte en omnívoros. Agregan que esta amplia gama de estrategias contribuyen a su gran abundancia en el mar, que también obedece a la gran producción de huevos y el rápido crecimiento asociado a la temperatura, y que se correlacionan más con el proto-zooplancton heterotrófico que con la clorofila- $a$, como fue este estudio; de forma que en sistema oligotróficos el consumo de ciliados puede equivaler al fitoplancton, a diferencia de sistemas mesotróficos, donde los ciliados son consumidos en menor proporción al fitoplancton. Este aspecto apoyaría en parte la correlación observada entre las altas abundancias de copépodos con las bajas concentraciones de clorofila- $a$.

En cuanto a la composición de la comunidad de copépodos puede decirse que buen porcentaje pertenecerían al orden Calanoida, común en varias áreas del Pacífico, por ejemplo en aguas de California y México
(Monsalve 1976, Farfán \& Álvarez 1992, Suárez-Morales 1994, Trujillo 1995, Smith \& Johnson 1997, Álvarez et al. 2003, Trujillo et al. 2004, López-Ibarra \& Palomares-García 2006, Aceves-Medina et al. 2007, Hernández-Trujillo et al. 2008), así como en el Caribe (Martínez-Barragán et al. 2009). Palomares et al. (1998), para el Pacífico mexicano reportaron que el 70\% de los copépodos clasificados son Calanoida y adicional determinaron que poco menos de la mitad de los adultos tienen tallas $<2 \mathrm{~mm}$.

\section{Agradecimientos}

Este trabajo fue posible gracias a la Vicerrectoría de Investigaciones de la Universidad Militar Nueva Granada por financiar el proyecto “Ictioplancton del Océano Pacífico Colombiano: prospección ecológica dentro del estudio regional del fenómeno El Niño. CIAS 835” y el apoyo logístico de la Dirección General Marítima (DIMAR), entidad que suministró el buque oceanográfico para la realización del crucero oceanográfico; su Centro Control de Contaminación del Pacífico (CCCP) suministró la información abiótica.

\section{LITERATURA CITADA}

Aceves-Medina G, G Esqueda-Escárcega, R Pacheco-Cavez, A Zárate-Villafranco, J Hernández-Alonso \& S Hernández-Trujillo. 2007. Cambios diarios en la composición y abundancia de copépodos planctónicos al sur de la Bahía de la Paz (Octubre de 2002). Hidrobiológica 17(2): 185-188.

Álvarez C, M Miranda \& G Lara. 2003. Familia Pontellidae (Crustacea: Copepoda) en la Bahía La Ventosa, Oaxaca, México: Sistemática y Ecología. Revista de Biología Tropical 51(3): 737-742.

Aridarni OH. 1990. Vertical distribution of calanoid copepods in the Banda Sea, Indonesia, during and after upwelling period. Bulletin of the Plankton Society of Japan 1 : 291-198.

Atencio F, L Gutiérrez \& S Gaviria. 2005. Copépodos planctónicos del complejo cenagoso de Malambo (Atlántico, Colombia) y su relación con algunos factores físicos y químicos del agua. Revista Dugandia 1(12): 17- 38.

Bernal A \& S Zea. 2000. Estructura de la comunidad de zooplancton en condiciones de descarga continental y de afloramiento costero en Santa Marta, Caribe Colombiano. Boletín de Investigaciones Marinas y Costeras 29: 3-26.

Calbet A \& E Saiz. 2005. The ciliate-copepod link in marine ecosystems. Aquatic Microbial Ecology 38: 157-167.

Carvajal LA, CF Vergara \& RH López. 2009. Chaetognatha, Thaliacea, Euphausiacea and pelagic Polychaeta in the Colombian Pacific Ocean during two periods in 1996 (La Niña) and two periods in 1997 (El Niño). Revista de la Facultad de Ciencias Básicas 5(1): 172-185. 
Cass CJ. 2011. A comparative study of eucalanoid copepods residing in different oxygen environments in the Eastern Tropical North Pacific: An emphasis on physiology and biochemistry. Tesis Ph. D., College of Marine Science, University of South Florida, Tampa, 154 pp.

CCCP. 2002. Compilación oceanográfica de la Cuenca Pacífica Colombiana. Centro Control de Contaminación del Pacífico, 109 pp. Ed. Imágenes de la Naturaleza, Cali.

Cervantes R, S Aguiñiga \& S Hernández. 1993. Condiciones de surgencia asociadas a la distribución de zooplancton en San Hipólito, B.C.S. Ciencias Marinas 19(1): 117-135.

Chen YQ. 1986. The vertical distribution of some pelagic copepods in the Eastern Tropical Pacific. CalCOFI Report 27: 205-227.

Clarke JA. 2008. An introduction to the dynamics of El Niño \& the southern oscillation, 308 pp. Academic Press, Londres.

Clesceri LS, AE Greenberg \& AD Eaton. 2001. Standard methods for the examination of water and wastewater, 1325 pp. American Public Health Association, Washington.

Farfán C \& S Álvarez. 1992. Biomasa del zooplancton del alto golfo de California. Ciencias Marinas 18(3): 17-36.

Fiedler PC \& LD Talley. 2006. Hydrography of the eastern tropical Pacific: A review. Progress in Oceanography 69: 143-180.

Fornes PA, BR Broitman, M Escobar \& P Báez. 2010. Composición de ácidos grasos en huevos y adultos de Tigriopus angulatus (Copepoda: Harpacticoida). Revista de Biología Marina y Oceanografía 45(3): 489-495.

Gaviria S \& N Aranguren. 2007. Especies de vida libre de la subclase Copepoda (Arthropoda, Crustacea) en aguas continentales de Colombia. Biota Colombiana 8(1): 53-68.

Giraldo A \& E Gutiérrez. 2007. Composición taxonómica del zooplancton superficial en el Pacífico colombiano (septiembre 2003) Investigaciones Marinas 35(1): 117-122.

Giraldo A, B Valencia \& DG Ramírez. 2011. Productividad planctónica y condiciones oceanográficas locales en Isla Gorgona durante julio 2006. Boletín de investigaciones Marinas y Costeras 40(1): 185-201.

Greenlanw C. 1979. Acoustical estimation of zooplankton populations. Limnology and Oceanography 24(2): 226-241.

Hernández-Trujillo S, A Zárate-Villafranco, R PachecoChávez, GM Esqueda-Escárcega, JR HernándezAlfonso \& G Aceves-Medina. 2008. Variación estacional de la producción de huevos del copépodo calanoideo Centropages furcatus (Dana, 1852) en la Bahía de La Paz, México. Hidrobiológica 18(1): 61-67.

Humphries S. 2007. Body size and suspension feeding. In: Hildrew AG, DG Raffaelli \& RE Brown (eds). Body size the estructure and function of aquatic ecosystems, pp. 1632. Cambridge University Press, Cambridge.

Landaeta MF, J Contreras \& CA Bustos. 2011. Fiordos de Chile: Potenciales zonas de crianza de Bathylagichthys parini (Pisces: Osmeriformes: Bathylagidae). Revista de Biología Marina y Oceanografía 46(1): 67-71.
Lavaniegos BE. 2007. El papel del zooplancton en la transferencia del carbono en el océano. En: Hernández B \& CG Gaxiola (eds). Carbono en ecosistemas acuáticos de México, pp. 129-140. Instituto Nacional de Ecología, México.

Lavaniegos B \& E González. 1999. Cambios en la comunidad de copépodos durante el ENSO 1992-93 en el canal de San Lorenzo, Golfo de California. Ciencias Marinas 25(2): 239265.

Lavens P \& P Sorgeloos. 1996. Manual on the production and use of live food for Aquaculture. FAO Fisheries Technical Paper 361: 1-295.

López-Ibarra G \& R Palomares-García. 2006. Estructura de la comunidad de copépodos en Bahía Magdalena, México, durante El Niño 1997-1998. Revista de Biología Marina y Oceanografía 41(1): 63-76.

López RH, CA López \& J Uribe. 2007. Quetognatos: ¿indicadores de eventos climáticos anómalos en el océano Pacífico colombiano? Boletín Científico CCCP 14: 109122. <http://dx.doi.org/10.1029/JC092iC13p14405>

Márquez B, J Díaz-Ramos, L Troccoli, B Marín \& R Varela. 2009. Densidad, biomasa y composición del zooplancton, en el estrato superficial de la cuenca de Cariaco, Venezuela. Revista de Biología Marina y Oceanografía 44(3): 737-749.

Martínez-Barragán MP, A Franco-Herrera, J MedinaCalderón \& A Santos-Martínez. 2009. La comunidad de copépodos en las islas de Providencia y Santa Catalina (Caribe Colombiano) durante el período lluvioso (octubre) 2005. Boletín de Investigaciones Marinas y Costeras 31(8): 85-103.

Medellín-Mora J \& GR Navas. 2010. Listado taxonómico de copépodos (Arthropoda: Crustacea) del mar Caribe colombiano. Boletín de Investigaciones Marinas y Costeras 39(2): 265-306.

Monsalve B. 1976. Copépodos del Pacífico colombiano, cruceros Pacífico V y VII. Divulgación Pesquera 18 (3/4): 2-9.

Paffenhöfer GA \& MG Mazzocchi1. 2003. Vertical distribution of subtropical epiplanktonic copepods. Journal of Plankton Research 25(9): 1139-1156.

Palomares R, E Suárez-Morales \& S Hernández-Trujillo. 1998. Catálogo de copépodos (Crustacea) pelágicos del Pacífico Mexicano. Catálogo de los copépodos (Crustacea) pelágicos del Pacífico Mexicano, 352 pp. ECOSURCICIMAR, México.

Pérez G, F Ramírez \& M Viñas. 1987. Variaciones de la abundancia numérica y biomasa del zooplancton de red en el golfo San Jorge (Año 1985). Revista de Desarrollo e Investigación Pesquera 7: 5-20.

Ramírez JG, A Franco-Herrera, D Rincón \& L Castro. 2005. Tasa de producción de fecas y coprofagía en Eucalanus subtenuis (Copepoda, Calanoida), bajo condiciones controladas de laboratorio. Boletín de Investigaciones Marinas y Costeras 34: 193-209.
Copepoda en aguas superficiales del Pacífico colombiano 
Rodríguez J. 2005. La estructura de tamaños del plancton: un tópico interdisciplinar y Margalefiano. Ecosistemas 14(1): 40-50.

Rodríguez-Rubio E \& W Schneider. 2003. On the seasonal circulation within the Panama Bight derived from satellite observations of wind, altimetry and sea surface temperature. Geophysical Research Letters 30(7): 1410.

Roman MR \& AL Gauzens. 1997. Copepod grazing in the Equatorial Pacific. Limnology and Oceanography 42(4): 623-634.

Saiz E, A Calbet \& E Broglio. 2003. Effects of small-scale turbulence on copepods: The case of Oithona davisae. Limnology and Oceanography 48(3): 1304-1311.

Siordia M, L Sánchez, M Sánchez \& M Franco. 2006. Variación temporal de la dieta de larvas de Bregmaceros bathymaster (Pisces; Bregmacerotidae) en las costas de Jalisco y Colima, México durante un ciclo anual (1996). Ciencias Marinas 32(1): 13-21.

Smith DL \& KB Johnson. 1997. A guide to marine coastal plankton and marine invertebrate larvae, $221 \mathrm{pp}$. Kendall / Hunt Publishing Company, Dubuque.

Smith P \& S Richardson. 1979. Técnicas modelo para prospecciones de huevos y larvas de peces pelágicos. FAO, Documento Técnico de Pesca 175: 1-107.

Suárez-Morales E. 1990. On the anomalous occurrence of bathypelagic calanoid copepods in surface waters off the Yucatan Peninsula. Plankton Newsletter 12(1): 4-7.

Suárez-Morales M. 1994. Copépodos planctónicos de la bahía de Chetumal, México (1990-1991). Caribbean Journal of Science 30(3-4): 181-188.

Takahashi T \& I Uchiyama. 2008. Seasonal changes in the density and vertical distribution of nauplii, copepodites and adults of the genera Oithona and Oncaea (Copepoda) in the surface water of Toyama Bay, southern Sea of Japan Plankton Benthos Research 3(3): 143-151.

Trujillo S. 1995. Cambios interanuales en la comunidad de copépodos de la costa occidental de baja California sur, México (Julio 1987 y julio 1988). Hidrobiológica 5(1-2): 127-134.

Trujillo SH, R Palomares, G López, G Esqueda \& R Pacheco. 2004. Riqueza específica de copépodos de Bahía Magdalena, Baja California Sur, México. Anales del Instituto de Biología 75(2): 253-270.
Turner JT. 2004. The importance of small planktonic copepods and their roles in pelagic marine food webs. Zoological Studies 43(2): 255-266.

Uribe HJ. 2003. Relaciones entre las condiciones ambientales y la comunidad fitoplanctónica (diatomeas y dinoflagelados), de la cuenca del Pacífico colombiano (1996-2001). Tesis de pregrado, Facultad de Biología Marina, Universidad Jorge Tadeo Lozano, Bogotá, 89 pp.

Velásquez A, J Rosas, T Cabrera, J Millán \& M Hernández. 2001. Efecto de Tetraselmis chuii, Nannochloris oculata y Dunaliella salina sobre el crecimiento poblacional de Apocyclops distans (Copepoda, Cyclopoidae) en diferentes condiciones de temperatura e iluminación. Revista de Biología Marina y Oceanografía 36(2): 189-197.

Vergara-Soto O, D Calliari, P Tiselius, R Escribano, L González \& S Soto-Mendoza. 2010. Functional response of Sagitta setosa (Chaetognatha) and Mnemiopsis leidyi (Ctenophora) under variable food concentration in the Gullmar fjord, Sweden. Revista de Biología Marina y Oceanografía 45(1): 35-42.

Villegas N \& I Málikov. 2006. Modelación de la estructura dinámica de las aguas de la cuenca del Pacífico colombiano. Boletín Científico CCCP 13: 97-114.

Woodward G \& P Warren. 2007. Body size and predatory interactions in freshwaters: scaling from individuals to communities. En: Hildrew AG, DG Raffaelli \& RE Brown (eds). Body size - the estructure and function of aquatic ecosystems, pp. 98-117. Cambridge University Press, Cambridge.

Woodward G, B Ebenman, M Emmerson, JM Montoya, JM Olesen, A Valido \& PH Warren. 2005. Body size in ecological networks. Trends in Ecology and Evolution 20(7): 402-409.

Wooster WS. 1959. Oceanographic observation in the Panama bight 'ASKOY' expedition, 1941. American Museum of Natural History 118 (3) : 113-152.

Wyrtki K. 1969. Surface currents of the eastern tropical Pacific Ocean. Inter-American Tropical Tuna Comision 9(5): 271303.

Zalkina A. 1970. Vertical distribution and diurnal migration of some Cyclopoida (Copepoda) in the tropical region of the Pacific Ocean. Marine Biology 5: 275-282. 